\title{
Coherence analysis of EEG in locomotion using graphs.
}

\section{Análisis de coherencia de señales EEG en locomoción usando grafos.}

\author{
G. Quiroz1, A. Espinoza-Valdez2, R.A. Salido-Ruiz2, L. Mercado1
}

"Universidad Autónoma de Nuevo León, FIME, Nicolás de los Garza, Nuevo León, México.

2Departamento de Ciencias Computacionales, CUCEI, Universidad de Guadalajara, Guadalajara, Jalisco, México.

\begin{abstract}
One of the most interesting brain machine interface (BMI) applications, is the control of assistive devices for rehabilitation of neuromotor pathologies. This means that assistive devices (prostheses, orthoses, or exoskeletons) are able to detect user motion intention, by the acquisition and interpretation of electroencephalographic (EEG) signals. Such interpretation is based on the time, frequency or space features of the EEG signals. For this reason, in this paper a coherence-based EEG study is proposed during locomotion that along with the graph theory allows to establish spatio-temporal parameters that are characteristic in this study. The results show that along with the temporal features of the signal it is possible to find spatial patterns in order to classify motion tasks of interest. In this manner, the connectivity analysis alongside graphs provides reliable information about the spatio-temporal characteristics of the neural activity, showing a dynamic pattern in the connectivity during locomotions tasks.
\end{abstract}

KEYWORDS: Coherence, feature extraction, graphs, EEG processing. 


\section{RESUMEN}

Una de las aplicaciones de las interfaces cerebro máquina (BMI, por las siglas en Inglés de brain machine interface) que en la actualidad han tenido mucho interés es el control de dispositivos de asistencia en rehabilitación de patologías neuromotrices. Esto es, que los dispositivos (prótesis, órtesis o exoesqueletos) tengan la capacidad de ejecutar la intención de movimiento del usuario, a través de la interpretación de las señales electroencefalográficas (EEG). Dicha interpretación se basa en el conocimiento de características en diferentes dominios de la señal EEG i.e., el dominio del tiempo, de la frecuencia o del espacio. Por tal motivo, en este trabajo proponemos un estudio sobre la coherencia de las señales EEG durante actividades de locomoción que, por medio de la teoría de grafos, nos permita establecer parámetros espacio-temporales característicos de las actividades motrices propuestas. Los resultados muestran que, además de las características temporales de la señal, es posible encontrar patrones espaciales que ayuden a clasificar las tareas motrices de interés. Esto es, el análisis de conectividad complementado con sus grafos asociados proporciona información confiable sobre las características espacio-temporales de la actividad neural, reflejando la dinámica de sus ajustes en correspondencia con distintos niveles de conectividad durante la marcha.

PALABRAS CLAVE: Coherencia, extracción de características, grafos, procesamiento de EEG.

\section{Correspondencia}

DESTINATARIO: Ricardo A. Salido-Ruiz

DIRECCIÓN: Blvd. Marcelino García Barragán \#1421,

Ciudad Universitaria, C.P. 44430, Guadalajara, Jal., México

CORREOELECTRÓNICO: drsalidoruiz@gmail.com

\section{Fecha de recepción:}

15 de octubre de 2016

Fecha de aceptación:

23 de diciembre de 2016 


\section{INTRODUCTION}

The main goal of brain machine interfaces (BMIs) is to provide a technological device in order to improve communication between brain and environment. According to the pathway of the neural information, BMIs are divided in efferent, afferent and bidirectional [1]. The first ones use neural information to decode motor intent, the extracted data is used afterwards to command an artificial actuator ${ }^{[2]}$. A efferent BMIs sense physical quantities from the environment and use such information to induce a stimulation in the brain (e.g. electrical microstimulation) [3,4]. Bidirectional BMIs are able to decode motor intention and encode sensory information to exchange it with the brain in a closed loop approach ${ }^{[5]}$. Nowadays BMIs are used in a wide variety of applications, from entertainment ${ }^{[6,7]}$ to therapeutics ${ }^{[8,9]}$. In the field of therapeutic applications, BMIs are assistive devices that enable patients with severe motor impairments to control external actuators such as prostheses, orthoses or exoskeletons ${ }^{[10]}$.

The restoration of locomotion in patients with lower-limb impairments using BCIs is a scientific and technological challenge. Currently one of the paradigms is to provide lower limb wearable robots for physical movement assistance and rehabilitation such as disabled people can recover their locomotion capabilities ${ }^{[11,12]}$. According to the efferent BMIs, the main idea is that the assistive device could be able to interpret the motion intention of the patient and carried it out. The understanding of the neural activity during human locomotion is the key for the interpretation of voluntary motion which has been widely studied in recent years. Two approaches have been proposed: (i) classification of time and/or frequency features from electroencephalographic (EEG) recordings ${ }^{[13,14]}$, and (ii) decoding of kinematic variables from the lower limbs from time-variant EEG frequency features ${ }^{[15-17]}$. Both schemes have been used to interpret EEG data mainly during well-controlled walking protocols ${ }^{[18-21]}$.
Although time and frequency features of EEG have provided useful information to understand neural activity, current advances in Neuroscience have pointed out that human locomotion involves a highly complex neural control in various locations of the central nervous system, including the planing and initiation in supraespinal areas (cortex, basal ganglia, midbrain and hindbrain) as well as timings and patterns of locomotor movements at spinal cord ${ }^{[22]}$. At this point emerges the interest in knowing the spatial behavior of the neural activity. This has two main purposes, to obtain information about the user motion intention and, allowing in this way that assistive devices can reproduce more precisely the human movement. In order to deal with spatial analysis of the neural activity in locomotion, in this work it is proposed a study of the neural connectivity during locomotion, including speed changes, in order to seek spatial patterns and their evolution that allows us to classify motion tasks.

Here, connectivity is studied through the coherence analysis, because is a widely used methodology in different fields of Neuroscience ${ }^{[23-25]}$, when functional connectivity between regions of the brain must be evaluated, and it can be useful to identify neuroanatomical and neurophysiological factors in EEG signals ${ }^{[26]}$. Also, the connectivity dynamics during locomotion is evaluated via the graphs associated to the respective coherence matrices. The paper is organized as follows. The experimental protocol along with the connectivity analysis is described in Methodology section. textcolorblueNext, connectivity results and their statistical analysis are presented in the Results section. Finally, in the last section a brief discussion and the main conclusions of this work are presented.

\section{METHODOLOGY Experimental Control}

A set of locomotion tasks were performed with three healthy research subjects, that did not present neuromotor pathologies in their lower limbs. All partici- 
pants gave their consent before the experiment. The experiment consisted in a controlled walk on a treadmill (XTERRA ${ }^{\circledR}$ trail racer 3.0) at two different velocities. The treadmill is set at velocity $1\left(V_{1}\right)$ and the subject start to walk; at this velocity ( $3 \mathrm{mph}$ ) the subjects should feel comfortable. After a minute in $V_{1}$ the treadmill velocity is changed to $V_{2}(5 \mathrm{mph})$. The transition time $\left(T_{1}\right)$ where an acceleration occurs is the time interval between $V_{1}$ and $V_{2}$. After a minute in $V_{2}$ the treadmill velocity changes back to $V_{1}$. The transition time $\left(T_{2}\right)$ where a deceleration occurs is the time between $V_{2}$ and $V_{1}$. Finally, the subject remains walking in $V_{1}$ for a minute. After that, the treadmill is stopped and the EEG acquisition is turned off after 15 second in idle state. This procedure was repeated ten times for each subject under examination, considering comfortable resting periods between trials.

The acquisition of the EEG signals was carried out by the B-Alert ${ }^{\circledR}$ X10 from Advanced Brain Monitoring, Inc. The system has nine electrodes according to the international 10-20 system (Fz, F3, F4, Cz, C3, C4, POz, $\mathrm{P} 3$ and $\mathrm{P} 4$ ), and its sampling frequency is $256 \mathrm{~Hz}$. The signal pre-processing (low and high frequencies noise filtering, and artifacts) was carried out by the inner filters of the acquisition system. Figure 1 shows the electrode distribution of the EEG equipment, according to the International 10-20 electrode placement system (left), and the research subject wearing the equipment (right). In order to study the neural connectivity, the spectral content of the EEG recordings at

$$
\gamma_{X Y}(\omega)=\frac{\left|S_{X Y}(\omega)\right|^{2}}{S_{X X}(\omega) S_{Y Y}(\omega)}
$$

where $S_{X X}$ and $S_{Y Y}$ define the auto-spectra densities of the $X$ and $Y$ time series, $S_{X Y}$ defines the cross-spectrum density of both signals. 
The result of Equation (1) is a set of coherence values in a frequency interval. When the coherence between electrodes is calculated, it is possible to group such sets in a symmetric matrix of dimension $n \times n$, where $n$ is the number of electrodes. In this case, the average coherence value is obtained among the range of the Nyquist frequency $\left(f_{s} / 2\right)$, where $f_{s}$ is the sample frequency:

$$
C=\frac{2}{f_{s}} \sum_{\omega=1}^{f_{s}} \gamma_{X Y}(\omega)
$$

In this manner, a symmetric matrix $C$ of dimension $n \times n$ is obtained, where $C$ coefficients determine the electrodes's interrelations. However, from $C$ the most important is know how relevant is the relation of each electrode respect to others. This is obtained by the average of the columns of $C$, i.e., $\bar{c}$ where their elements are given by:

$$
\bar{c}_{i}=\frac{1}{n-1} \sum_{\substack{j=1 \\ j \neq i}}^{n} c_{i j},
$$

where $\bar{c}_{i}$ is a weighted vector that determines the relative importance of the electrode in the interconnection, thus it represents the average connectivity degree between electrodes and $c_{i j}$ are the elements of $C$.

In order to analyze the statistical data of the connectivity, an intra-subject one-way ANOVA was carried out by Minitab ${ }^{\mathrm{R}}$ 17. The analysis was organized as follows: the connectivity coefficient of the $n$ electrodes per subject, three velocities $\left(V_{1}, V_{2}\right.$ and $\left.V_{1}\right)$ and two transition $\left(T_{1}\right.$ and $\left.T_{2}\right)$ stages that were repeated 10 times by each subject. The null hypothesis considers that the five stages means are equal, while in the alternative hypothesis considers that at least one mean is different from the others. The significance level are set at $\alpha=0.05$. The same assumptions made for meas are considered to analyze the variance of variables among the trials.

\section{Graphs}

The coherence is analyzed in pairs of electrodes, this is, the coherence value represents the connection weight between them. Therefore, the matrix $C$ can be represented by a connectivity graph. According to the Graph Theory, a graph $G$ is an ordered triad $(V, E, \Psi)$ which consist of a not empty set $V$ of vertices, an $E$ set of edges, and an incidence function $\Psi: E \rightarrow V$. For each edge the following condition holds: $\Psi$ associates the edge $e$ to a pair of vertices of $V, \Psi(e)=u, v$ [29]. In this work the adjacent matrix of a graph $G$ with a set of vertices $V$ (electrodes) is the square matrix $C=C(G)$ of dimension $n \times n$. Each element $c_{i j}$ of this matrix ranges in $[0.5,1]$ if $v_{i}, v_{j}$ belongs to $E$, elsewhere zero. Matrix $C$ is symmetric with the null diagonal. The grade of vertex $v_{i}$ is $d\left(v_{i}\right)$, and it is defined as the number of $c_{i j} \geq 0,5$ in each row (or column). 


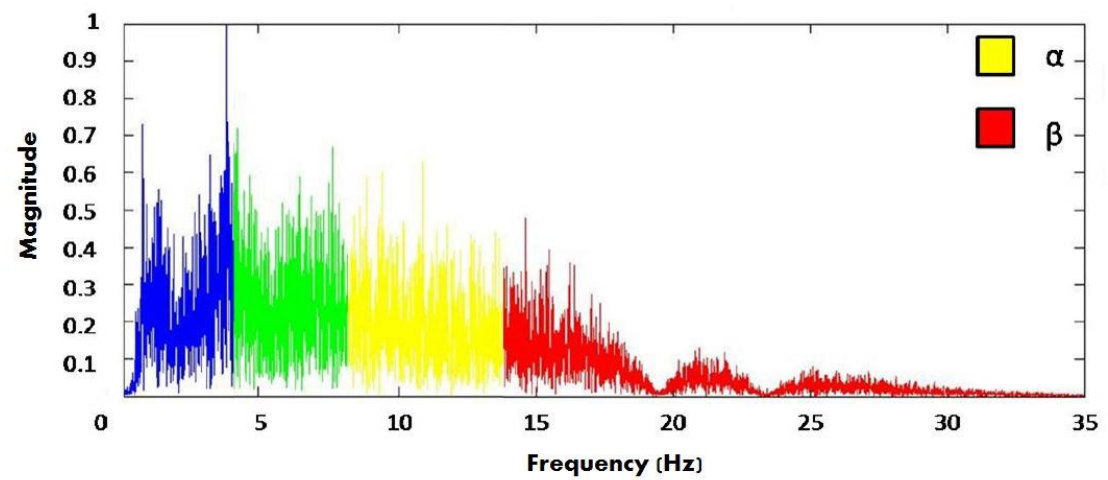

FIGURE 2: Frequency spectrum of a neural signal. The $\alpha(8-13 \mathrm{~Hz})$ and $\beta(13-30 \mathrm{~Hz})$ bands provides information about the neural activity during motion activities [27].

The one-way ANOVA results ( $F$ and $p$ values) for the three research subjects are the following: Subject $1(F=11.31$, $p \approx 0.000)$, Subject $2(F=9.84, p \approx 0.000)$ and Subject $3(F=16.47, p \approx 0.000)$. This implies that significant differences (inside the $95 \%$ Confidence Interval) were found among the means of velocity $\left(V_{1}, V_{2}\right.$ and $\left.V_{1}\right)$ and transition stages ( $T_{1}$ and $T_{2}$ ) at each subject. The statistical parameters of the coherence in $\alpha$-band are summarized in Table 1 , whereas Table 2 shows the statistical parameters of coherence in $\beta$-band. In both tables, the number of samples is defined as the average connectivity degree computed from data of $\alpha$ and $\beta$ bands for each motion task.

\begin{tabular}{|c|c|c|c|c|c|}
\hline & Motion tasks & Number of samples & Mean & Standard deviation & $95 \%$ CI \\
\hline Subject 1 & $V_{1}$ & 90 & 3.8438 & 0.8514 & $(3.6721,4.0154)$ \\
& $T_{1}$ & 90 & 4.2785 & 0.7974 & $(4.1068,4.4501)$ \\
& $V_{2}$ & 90 & 3.7690 & 0.8827 & $(3.5973,3.9406)$ \\
& $T_{2}$ & 90 & 4.4033 & 0.7636 & $(4.2317,4.5750)$ \\
& $V_{1}$ & 90 & 3.8267 & 0.8426 & $(3.6550,3.9983)$ \\
\hline Subject 2 & $V_{1}$ & 90 & 4.4834 & 0.6418 & $(4.3463,4.6204)$ \\
& $T_{1}$ & 90 & 4.0492 & 0.7346 & $(3.9121,4.1862)$ \\
& $V_{2}$ & 90 & 4.5728 & 0.6377 & $(4.4358,4.7098)$ \\
& $T_{2}$ & 90 & 4.1694 & 0.6308 & $(4.0324,4.3065)$ \\
& $V_{1}$ & 90 & 4.3952 & 0.6572 & $(4.2582,4.5323)$ \\
\hline Subject 3 & $V_{1}$ & 90 & 4.2568 & 0.7842 & $(4.0902,4.4235)$ \\
& $T_{1}$ & 90 & 4.4280 & 0.7987 & $(4.2614,4.5946)$ \\
& $V_{2}$ & 90 & 3.5807 & 0.8662 & $(3.4141,3.7473)$ \\
& $T_{2}$ & 90 & 3.8268 & 0.7687 & $(3.6602,3.9934)$ \\
& $V_{1}$ & 90 & 4.1831 & 0.7998 & $(4.0164,4.3497)$ \\
\hline
\end{tabular}

\section{TABLE 1: Results from statistical analysis of $c$ in $\alpha$-band: mean, standard deviation and confidence} interval intra-subjects (CI).

Additional to the results presented in Tables 1 and 2, Figure 3, 4 and 5 show the average and standard deviation of the filtered signals in the $\alpha$-band (A) and $\beta$-band (B) for each of the three research subjects. In these figures, the dark-blue boxes include the means and variance of the average connectivity degree of each of the nine electrodes $\bar{c}_{i}$ (horizontal axes) in each motion task described in the experimental protocol. More precisely, the first light-blue box represents velocity $1\left(V_{1}\right)$, the second light-blue box is velocity $2\left(V_{2}\right)$, whereas the third light-blue box is the $V_{1}$ again. The transition between $V_{1}$ and $V_{2}$ is the first white box $\left(T_{1}\right)$ and the transition between $V_{2}$ and $V_{1}$ is the second white box $\left(T_{2}\right)$. 


\section{Electrodes connectivity}

A graph was construct in order to observe the connectivity dynamics between electrodes, during the motion tasks (both, velocities and transitions). Based on the statistical analysis of the intra-subject result, an average graph at each of the five motion tasks $\left(V_{1}, T_{1}, V_{2}, T_{2} V_{1}\right)$, considering the three research subject and the ten performed trials. Figure 6 shows the topological structure of such graphs considering $\alpha$-band of the EEG signal of each electrode. On the Figure 7 the graphs of $\beta$-band are shown.

\section{DISCUSSION}

As it was presented in Figure 3.A, Figure 4.A, Figure 5.A and Tables 1 and 2, the means of the motion tasks differ among them significantly; most precisely, means of velocity stages differ from means of transition stages while means of the same-kind stage (velocity/transition) do not differ in Subject 1 and 2, i.e. means are very close. A particular behavior is presented in Subject

G. Quiroz et al. Coherence analysis of EEG in locomotion using graphs

\begin{tabular}{|l|c|c|c|c|c|}
\hline & Motion tasks & Number of samples & Mean & Standard deviation & $95 \%$ CI \\
\hline Subject 1 & $V_{1}$ & 90 & 4.7361 & 0.7839 & $(4.5777,4.8945)$ \\
& $T_{1}$ & 90 & 4.9975 & 0.7760 & $(4.8391,5.1559)$ \\
& $V_{2}$ & 90 & 4.7691 & 0.8005 & $(4.6107,4.9275)$ \\
& $T_{2}$ & 90 & 5.1683 & 0.6629 & $(5.0099,5.3267)$ \\
& $V_{1}$ & 90 & 4.6481 & 0.7913 & $(4.4897,4.8065)$ \\
\hline Subject 2 & $V_{1}$ & 90 & 4.1777 & 0.7842 & $(4.0076,4.3479)$ \\
& $T_{1}$ & 90 & 4.0126 & 0.8694 & $(3.8424,4.1827)$ \\
& $V_{2}$ & 90 & 4.4906 & 0.8042 & $(4.3205,4.6608)$ \\
& $T_{2}$ & 90 & 4.1439 & 0.8003 & $(3.9737,4.3140)$ \\
& $V_{1}$ & 90 & 4.0700 & 0.8455 & $(3.8999,4.2402)$ \\
\hline Subject 3 & $V_{1}$ & 90 & 4.4796 & 0.8673 & $(4.3001,4.6590)$ \\
& $T_{1}$ & 90 & 4.4056 & 0.8576 & $(4.2261,4.5850)$ \\
& $V_{2}$ & 90 & 4.3494 & 0.9073 & $(4.1700,4.5289)$ \\
& $T_{2}$ & 90 & 4.3984 & 0.8435 & $(4.2189,4.5778)$ \\
& $V_{1}$ & 90 & 4.5531 & 0.8542 & $(4.3737,4.7326)$ \\
\hline
\end{tabular}

TABLE 2: Results from statistical analysis of $\boldsymbol{c}$ in $\beta$-band: mean, standard deviation and confidence interval intra-subjects (Cl).

3, where also significant differences between means of the same stage are present i.e. $V_{2}$ mean differs from both inicial $V_{1}$ and final $V_{1}$ means while $T_{1}$ mean differs from $T_{2}$ mean.

In general, an increment in the neural activity is reported (by the suppression of activity in $\alpha$ and $\beta$ bands) over the cortical sensorimotor areas, which correspond to the lower limb control areas during locomotion, in comparison to imagery locomotion. On the other hand, there is a suppression of the $\alpha$ and $\beta$ bands in the premotor and sensorimotor areas during locomotion, contrary to resting state such as it was previously reported in $[18,20]$.

From the statistics of the coherence analysis, it can be shown that the $\alpha$-band (Figure 3.A, Figure 4.A and Figure 5.A) provides more information than the $\beta$-band (Figure 3.B, Figure 4.B and Figure 5.B) regarding connectivity dynamics in each of the motion tasks. Figures 3-5 show that the electrode $C_{z}$ is the most reliable in all the activities, preserving its behavior between the three research subjects. To identify a reliable electrode gives the possibility of 
use its data as command control in BMIs applications. Regarding the spatial analysis of the connectivity, Figures 6 and 7 show that during the transitions the connectivity between electrodes tends to increase, while the connectivity during speed periods decreases. Moreover, the topology of $V_{1}$ is preserved independently disregarding which transition occurred. Although during $V_{2}$ the connection pattern holds, the connectivity grade increases in both bands (see Figure 6 and 7). These results are in concordance with the literature regarding EEG features during locomotion, but the graph dynamics shows the neural connectivity changes.

\section{CONCLUSION}

The use of the neural information from EEG signals is useful in BMIs application to interpret user motion intention. In this work it is shown that, along the frequency features of the signal, it is possible to find spatial patterns to classify locomotion tasks. In this manner, the connectivity analysis alongside graphs provides reliable information about the spatio-temporal characteristics in the neural activity, where dynamical evolution of the pattern during locomotions tasks is shown (see Figure 6 and 7). For BMIs applications it is important to find patterns that hold between

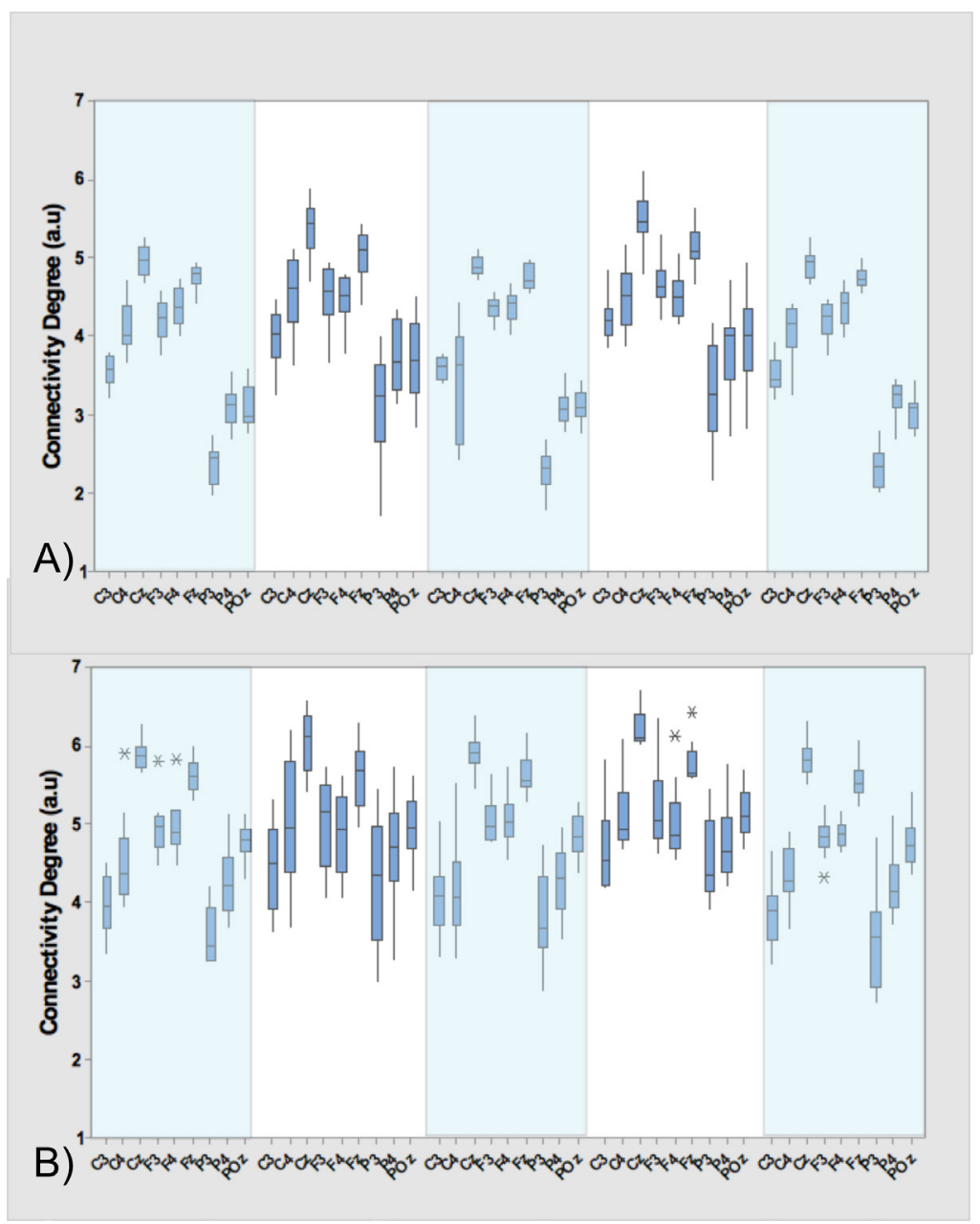

FIGURE 3: Analysis of data of Subject 1. Mean and standard deviation of $c_{i}$ during the motion tasks, that is, velocities $V_{1}, V_{2}$ (dark-blue boxes) and transitions $T_{1}$ and $T_{2}$ (light-blue boxes). The analysis includes $\alpha(A)$ and $\beta$ (B) bands. 
subjects, as it was shown in this work. The role of the subject differences, as well as to include more variables in the experimental protocol such as treadmill angle and more walking speeds would be of interest in future works.

In general we have observed that differences among motion tasks can be detected from connectivity coeffcients such as coherence. The connectivity patterns in $V_{1}, V_{2}, T_{1}$ and $T_{2}$ are spatial characteristics of the neural activities for these well-defined locomotion tasks, and they can be quantified and validated by the statistical analysis of the average of the connectivity degree $(c)$ along the full-locomotion trail, which includes the five motions tasks. Thus, the results also provide the time-evolution of the characterized spatial patterns. The proposed approach provides a quantitative analysis to find differences among motion tasks in order to show that is possible to use them as inputs of a BMI system.

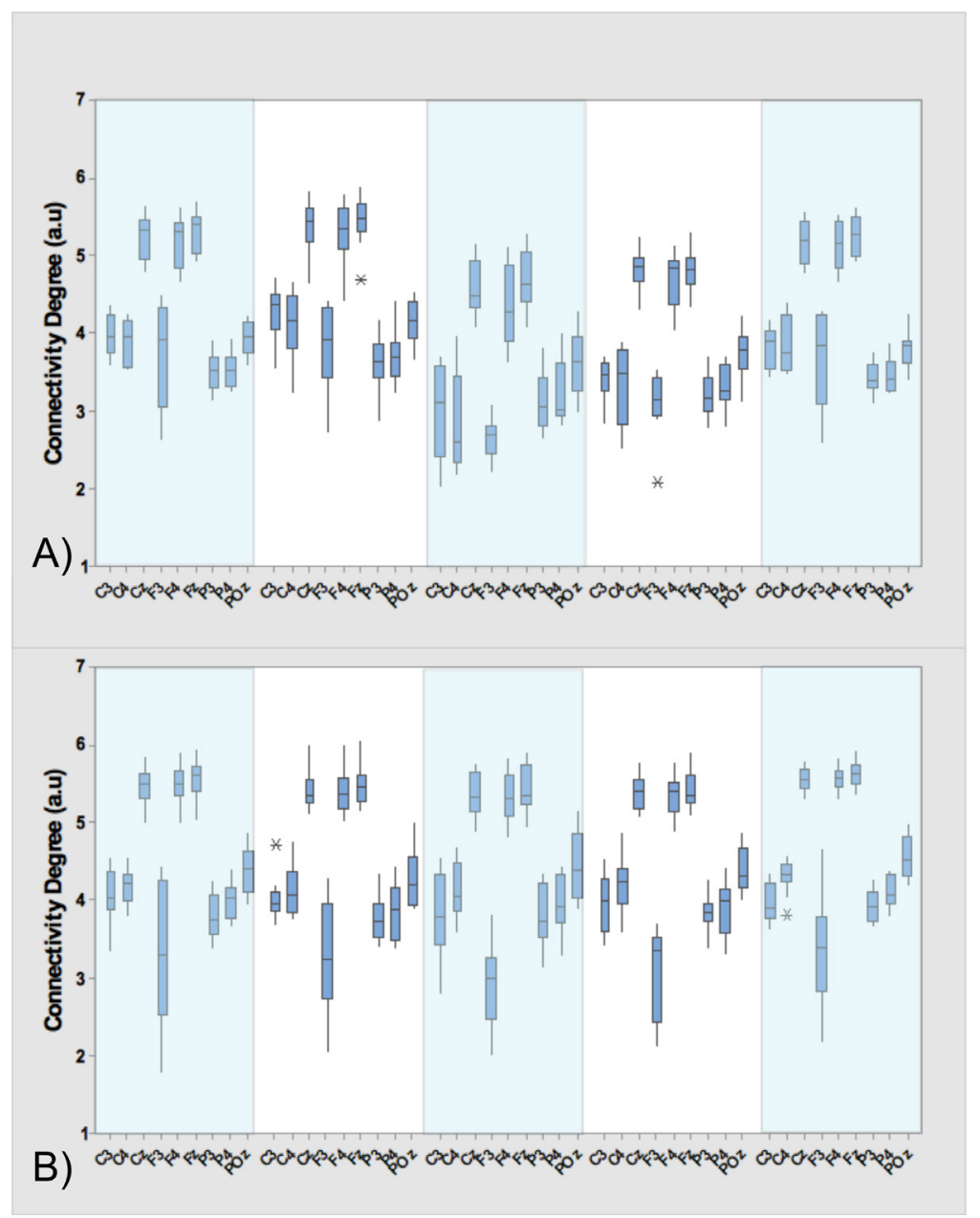

FIGURE 4: Analysis of data of Subject 2. mean and standard deviation of $c_{i}$ during the motion tasks, that is, velocities $V_{1}, V_{2}$ (dark-blue boxes) and transitions $T_{1}$ and $T_{2}$ (light-blue boxes). The analysis includes $\alpha(A)$ and $\beta$ (B) bands. 


\section{ACKNOWLEDGMENTS}

Authors are members of the research network "Biosystems and Biomechatronics" and thank to PRODEP-SEP for financial support by the projects 103.5/15/14156 y P3e231277. Luis Mercado thanks to CONACYT for granting the scholarship number 401834. G. Quiroz thanks to PAICYT-UANL for grant IT437-15.

\section{COMPETING INTERESTS}

The authors declare that they have no competing interests.

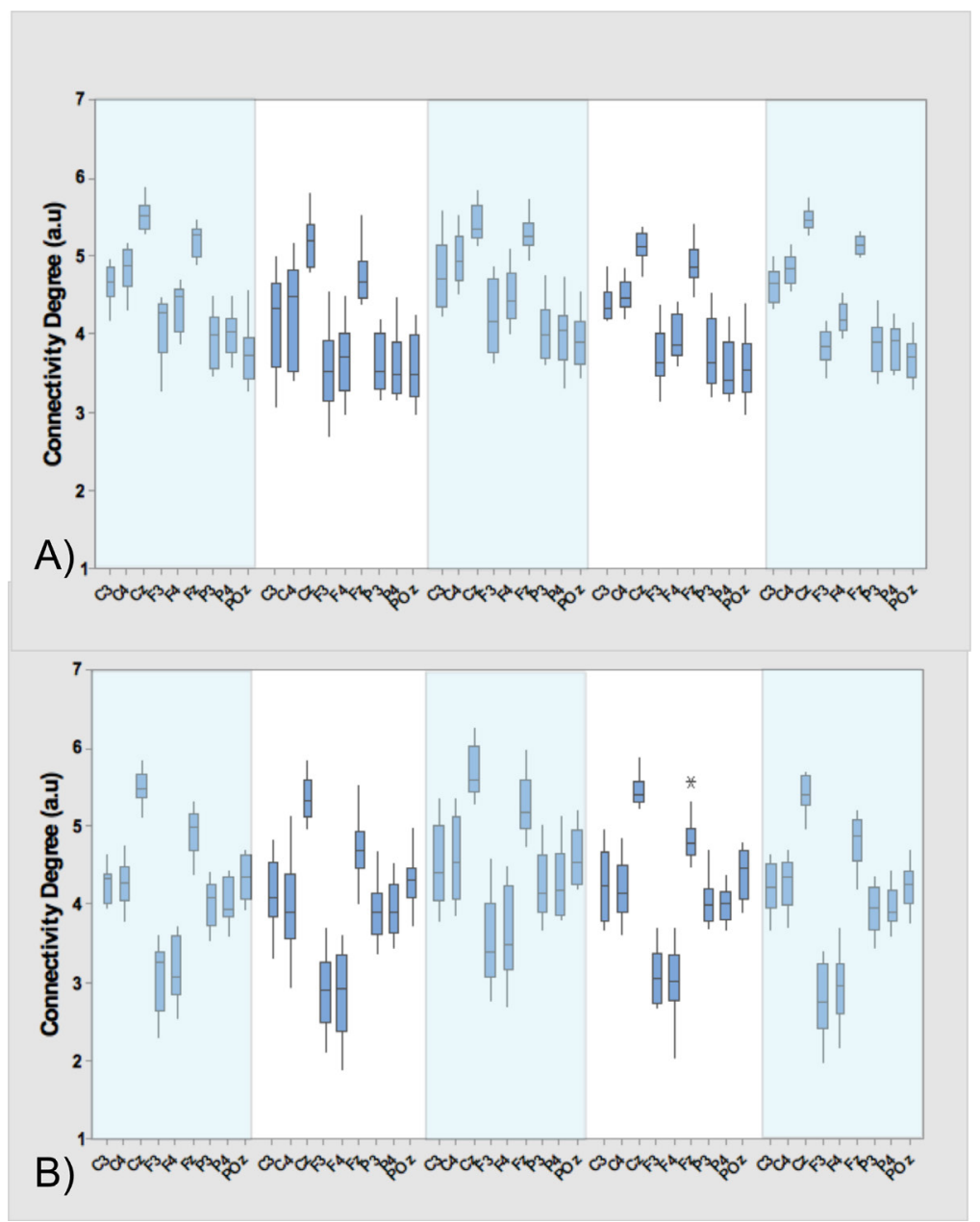

FIGURE 5: Analysis of data of Subject 3. mean and standard deviation of $c_{i}$ during the motion tasks, that is, velocities $\boldsymbol{V}_{1}, \boldsymbol{V}_{2}$ (dark-blue boxes) and transitions $T_{1}$ and $T_{2}$ (light-blue boxes). The analysis includes $\alpha(A)$ and $\beta$ (B) bands. 


\section{LIST OF REFERENCES}

[1] Panzeri S, Safaai H, De Feo V, Vato A, "Implications of the dependence of neuronal activity on neural network states for the design of brain-machine interfaces”, Frontiers in Neuroscience, vol. 10, pp. 1-8, 2016.

[2] Donoghue JP, “Connecting cortex to machines: recent advances in brain interfaces”, Nature Neuroscience Supplement, vol. 5, pp. 1085-1088, 2002.

[3] Fitzsimmons NA, Drake W, Hanson TL, Lebedev MA, Nicolelis MAL, "Primate reaching cued by multichannel spatiotemporal cortical microstimulation”, Journal of Neuroscience, vol. 23, pp. 5593-5602, 2007.

[4] Alam M, Rodrigues W Pham BN, Thakor NV, "Brain-machine interface facilitated neurorehabilitation via spinal stimulation after spinal cord injury: recent progress and future perspectives”, Brain Research, vol. 1646, pp. 25-533, 2016.
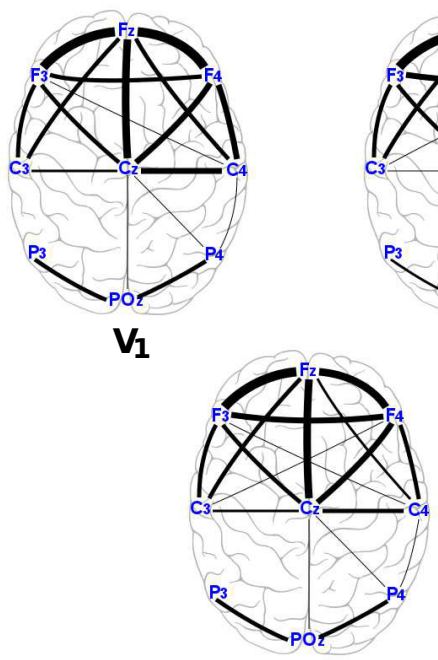

$\mathbf{T}_{1}$
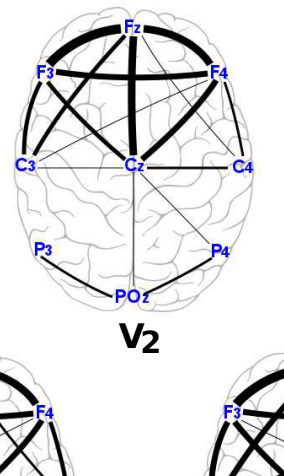

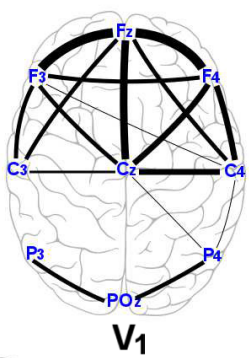

$\mathrm{V}_{1}$

\section{FIGURE 6: Connectivity graphs of the motion tasks for the average date of the three research subjects in $\alpha$-band.}

Moxon KA, Foffani G, “Brain-machine interfaces beyond neuroprosthetics”, Neuron, vol. 86, pp. 55-67, 2015.

Liao L, Chen C, Wang I, "Gaming control using a wearable and wireless EEG-based brain-computer interface device with novel dry foam-based sensors”, Journal of Neuroengineering Rehabilitation, vol. 9, pp. 5, 2012.

Park JL, Fairweather MM, Donaldson DI, “Making the case for mobilecognition: EEG and sports performance”, Neurosciences Biobehavior Reviews, vol. 52, pp. 117-130, 2015.

Wright J, Macefield VG, van Schaik A, Tapson JC, “A review of control strategies in closed-loop neuroprosthetic systems ”, Frontiers in Neuroscience, vol. 10, pp. 1-13, 2016.

Millán JdR, Rupp R, Müeller-Putz GR, Murray-Smith R, Giugliemma C, Tangermann M, et al., “Combining brain-computer interfaces and assistive Technologies: State-of-the-art and challenges”, Frontiers in Neuroscience, doi:10.3389/fnins.2010.00161, 2010.

Chaudhary U, Birbaumer N, Ramos-M
doi:10.1038nrneurol.2016.113, 2016.

Huo W, Mohammed S, Moreno JC, Amirat Y, “Lower limb wearable robots for assistance and rehabilitation: a state of the art”, IEEE systems Journal, vol. 10, pp. 1068-1081, 2016.

Onose G, Cardei V, Craciunoiu ST, Avramescu V, Opris I, Lebedev MA, Constantinescu MV, "Mechatronic wearable exoskeletons for bionic bipedal standing and walking: a new synthetic approach”, Frontiers in Neuroscience, vol. 10, pp. 1-9, 2016. 


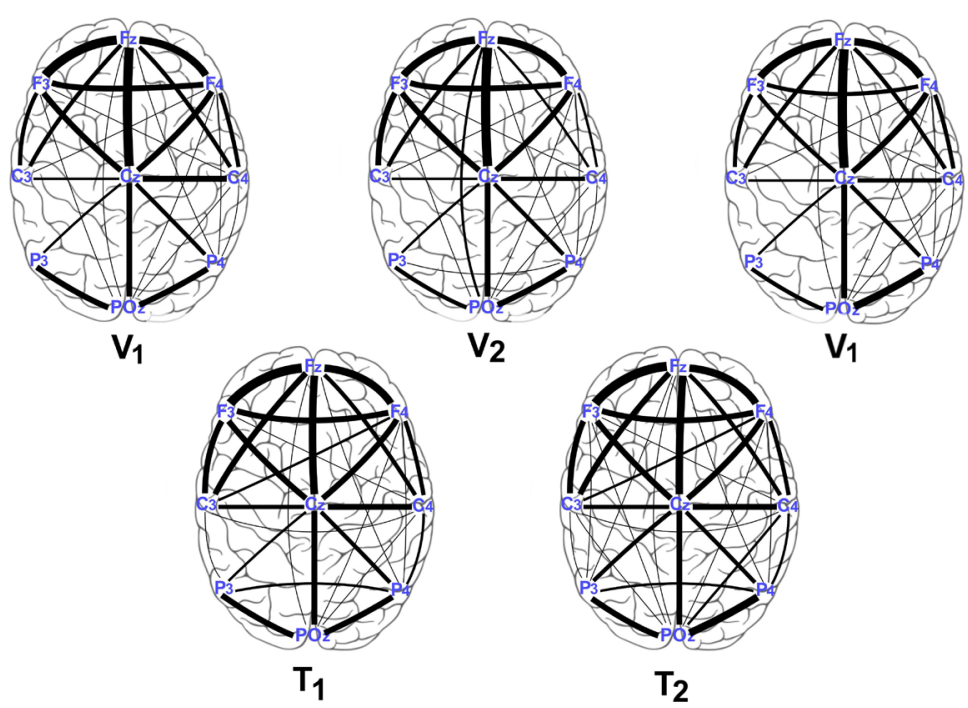

FIGURE 7: Average connectivity graphs in the $\beta$-band during the motion tasks.

[13] Lin BS, PanJS, Chu TY, Lin BS, "Development of a wearable motor-imagery-based brain-computer interface”, Journal of Medical Systems, vol. 40, pp. 71, 2016.

[14] Huan NJ, Palaniappan R, "Neural network classification of autoregressive features from electroencephalogram signals for brain computer interface design”, Journal of Neural Engineering, vol. 1, no. 142, pp. 150, 2004.

[15] Phat Luu T, He Y, Brown S, Nakagame S, Contreras-Vidal JL, “Gait adaptation to visual kinematic perturbations using a real-time closed-loop braincomputer interface to a virtual reality avatar”, Journal of Neural Engineering, vol. 13, no. 3, pp. 1-13, 2016.

[16] Ubeda A, Planelles D, Costa A, Hortal E, Iáñez E, Azorín JM, “Decoding knee angles from EEG signals for di erent walking speeds”, 2014 IEEE International Conference on Systems, Man, and Cybernetics (SMC), pp. 1475-1478, 2014.

[17] Presacco A, Forrester LW, Contreras-Vidal JL, “Decoding Intra-Limb and Inter-Limb Kinematics During Treadmill Walking From Scalp Electroencephalographic (EEG) Signals”, IEEE Transactions on Neural Systems Rehabilitation Engineering, vol. 20, no. 2, pp. 212-219, 2012.

[18] Wagner J, Solis-Escalante T, Grieshofer P, Neuper C, Müller-Putz G, Scherer R, "Level of participation in robotic-assisted treadmill walking modulates midline sensorimotor EEG rhythms in able-bodied subjects”, Neuroimage, vol. 63, no. 3, pp. 1203-1211, 2012.

[19] King CE, Wang PT, Chui LA, Do AH, Nenadic Z, "Operation of a brain-computer interface walking simulator for individuals with spinal cord injury”, Journal of NeuroEngineering and Rehabilitation, vol. 10, pp. 1-14, 2013.'

[20] Wagner J, Solis-Escalante T, Scherer R, Neuper C, Müller-Putz G. "It’s how you get there: walking down a virtual alley activates premotor and parietal areas", Frontiers in Human Neuroscience, vol. 8, no. 93, 2014.

[21] Castermans T, Duvinage M, Cheron G, Dutoit T, "About the cortical origin of the low-delta and high-gamma rhythms observed in EEG signals during treadmill walking”, Neuroscience Letters, vol. 561, pp. 166-70. 2014.

[22] Kiehn O, "Decoding the organization of spinal circuits that control locomotion”, Nature Reviews of Neurosciences, vol. 17, no. 4, pp. 224-238, 2016.

[23] Schwartz S, Kessler R, Gaughan T, Buckley AW, "EEG coherence patterns in autism: an updated review”, Pediatric Neurology, doi 10.1016/j.pediatrneurol.2016.10.018, 2016.

[24] Teramoto H, Morita A, Ninomiya S, Akimoto T, Shiota H, Kamei S, "Relation between resting state front-parietal EEG coherence and executive function in parkinson's disease”, BioMED Research International, vol. 2016, pp. 1-6, 2016.

[25] Kühn-Popp N, Kristen S, Paulus M, Meinhardt J, Sodian B, "Left hemisphere EEG coherence in infancy predicts infant declarative pointing and preschool epistemic language”, Social Neuroscience, vol. 11, pp. 49-59, 2015.

[26] Chorlian DB, Rangaswamy M, Porjesz B, “EEG coherence: topography and frequency structure”, Exp Brain Res vol. 198, pp. 59-83, 2009.

[27] Proakis JG, Manolakis DG Digital signal processing. Third Edition. Prentice-Hall, New Jersey, 1996.

[28] Nunez PL, Silberstein RB, Shi Z, Carpenter MR, Srinivasan R, Tucker DM, et al., "EEG coherency II: experimental comparisons of multiple measures", Clin Neurophysiol, vol. 110, no. 3, pp. 469-486, 1999.

[29] Diestel R, Graph Theory. Springer-Verlag Heidelberg, New York, 2005. 